\title{
Eficacia de fenilefrina en la prevención de hipotensión en cesárea bajo anestesia subaracnoidea
}

\author{
Nelly Johanna Puentes-Vega* \\ Héctor Julio Meléndez-Flórez** \\ Jorge de Lujan Alvarado-Arteaga***
}

\begin{abstract}
* Médica. Residente de III año de Anestesiología y reanimación. Universidad Industrial de Santander. Bucaramanga. Santander. Colombia.
**Médico. Anestesiólogo. Intensivista. Epidemiólogo. Profesor titular. Departamento de cirugía. Universidad Industrial de Santander. Bucaramanga. Santander. Colombia.

***Médico. Anestesiólogo. Coordinador cirugía, Clínica Materno Infantil San Luis. Bucaramanga. Santander. Colombia.

Correspondencia: Dra. Nelly Johanna Puentes Vega. Dirección: Calle 20 \#30-27 Edificio Platinium. Torre 1 Apto 604. Bucaramanga. Santander. Colombia. Teléfono: 301-2518679 Correo electrónico: puentesjohanna@gmail.com
\end{abstract}

\section{Resumen}

Introducción: la hipotensión es el evento adverso más incidente cuando se administra anestesia subaracnoidea para cesárea; múltiples estrategias para su prevención y manejo se han propuesto, entre ellas el uso de vasopresores profilácticos con resultados no concluyentes. Objetivo: evaluar la eficacia preventiva del uso de Fenilefrina en la hipotensión arterial inducida por la anestesia subaracnoidea en gestantes llevadas a cesárea. Materiales y Métodos: ensayo clínico controlado, doble ciego, en gestantes, mayores de 18 años, ASA 1 y 2, quienes recibieron anestesia subaracnoidea para cesárea, fueron aleatorizadas a recibir placebo o infusión profiláctica de fenilefrina $40 \mathrm{mcg} / \mathrm{min}$, durante 5 minutos. Resultados: se incluyeron 140 pacientes en el análisis de datos. La incidencia de hipotensión fue mayor en el grupo control que en el intervenido (RA 45,71\% vs 32,85\%). Sin presentarse diferencias significativas entre ambos grupos ( $p=0,119$ ). RR de hipotensión en el grupo intervenido fueron menores, independiente del criterio de hipotensión (tensión arterial sistólica, RR 0,72 (IC95\% 0,47-1,09); tensión arterial media, RR 0.93 (IC95\% 0,64-1,37); TAS o TAM RR 0.86 (IC95\% 0,62-1,21)), pero sin diferencias significativas $p>0,05$. El uso de vasopresores en los pacientes que presentaron este evento se dio en $75.4 \%(n=52)$, con RR mayor en el grupo control y diferencias significativas al evaluar los dos grupos. (RR: 1,4 p=0,032). Conclusiones: fenilefrina profiláctica no demostró ser eficaz en disminuir la incidencia de hipotensión; sin embargo, sí se asocia con una disminución significativa en los requerimientos de vasopresores, así como en la incidencia de náuseas y vómito. MÉD.UIS. 2018;31(2):33-9.

Palabras clave: Cesárea. Anestesia Raquídea. Hipotensión. Fenilefrina

\section{Effectiveness of phenylefrine in prevention of hypotension during spinal anesthesia for cesarean delivery}

\section{Abstract}

Background: Hypotension is the most common adverse event when subarachnoid anesthesia is administered for cesarean section; multiple strategies for its prevention and management have been proposed, including the use of prophylactic vasopressors with inconclusive results. Objective: To evaluate the preventive efficacy of the use of phenylephrine in hypotension induced by subarachnoid anesthesia in pregnant women taken to caesarean section. Methods: A double-blind, controlled clinical trial in pregnant women, older than 18 years old, ASA 1 and 2, who received a subarachnoid anesthesia for cesarean section, were randomized to receive placebo or prophylactic infusion of phenylephrine $40 \mathrm{mcg} / \mathrm{min}$, for 5 minutes. Results: 140 patients were included in data analysis. Incidence of hypotension was higher in the control group than in the intervention group (AR $45.71 \%$ vs $32.85 \%$ ). There were no significant differences between the two groups ( $p$ $=0.119$ ). RR of hypotension in the intervention group were lower, independent of the criterion of hypotension [systolic blood pressure, RR 0.72 (95\% Cl 0.47-1.09), mean arterial pressure, RR 0.93 ( $95 \% \mathrm{Cl} 0.64-1.37$ ), SBP or MAP 0.86 (RR 0.86) (IC95\% 0.62-1.21)], but without significant differences $p>0.05$. The use of vasopressors in patients who presented this event occurred in $75.4 \%(n=52)$, with higher RR in

Artículo recibido el día 18 de mayo de 2017 y aceptado para publicación el 13 de marzo de 2018 
the control group and significant differences when evaluating the two groups. (RR 1.4 P = 0.032). Conclusions: Prophylactic phenylephrine did not prove effective in reducing the incidence of hypotension, however, it is associated with a significant decrease in vasopressor requirements, as well as in the incidence of nausea and vomiting. MÉD.UIS. 2018;31(2):33-9.

Keywords: Cesarean Section. Spinal anesthesia. Hypotension. Phenylephrine.

¿Cómo citar este artículo?: Puentes-Vega NJ, Meléndez-Flórez HJ, Alvarado-Arteaga JL. Eficacia de fenilefrina en la prevención de hipotensión en cesárea bajo anestesia subaracnoidea. MÉD. UIS. 2018;31(2):33-9. doi: 10.18273/revmed.v31n2-2018004

\section{Introducción}

En 1985, la Organización Mundial de la Salud (OMS) propuso que del total de los nacimientos, el porcentaje de cesáreas debería encontrarse entre 10 y $15 \%$. Sin embargo, en las últimas dos décadas, se ha evidenciado un incremento en la frecuencia de cesáreas. En Colombia, la tendencia a la realización de cesáreas presenta un comportamiento similar al mundial, reportándose un incremento en su tasa durante los últimos años del $24,9 \%$ en 1998 a $45,7 \%$ en $2013^{2}$. Diversos estudios han demostrado que el origen de este incremento es multifactorial ${ }^{3}$.

Por ende, el anestesiólogo se ha visto en la necesidad de enfrentarse a este procedimiento, lo que ha generado una constante búsqueda de la mejor técnica anestésica. Teniendo en cuenta la dificultad en el manejo de la vía aérea de la materna, la necesidad de disminuir la exposición del feto a los medicamentos con el fin de evitar la depresión neonatal y la posibilidad de la madre de permanecer despierta y disfrutar de la experiencia del nacimiento, se ha llegado a considerar el empleo de la anestesia neuroaxial, permitiendo mejorías en su calidad con la adición de opioides al anestésico local, disminución de la perdida sanguínea, así como también una reducción en la mortalidad materna comparado con las técnicas generales ${ }^{4,5}$.

Dentro del estudio de esta técnica anestésica, se ha documentado la hipotensión arterial como la complicación más frecuente. Esta se acompaña generalmente de náuseas o vómito y alteración del estado de conciencia, generando un riesgo importante para la madre de aspiración pulmonar; también se puede asociar a disnea, isquemia de órganos o incluso colapso cardiovascular con la consecuente alteración en la perfusión placentaria, provocando hipoxia, acidosis y posible lesión neurológica en el feto 4 .
Según estudios realizados, la incidencia reportada de hipotensión varía desde el 7,4\% hasta un 90\%, encontrándose esta gran diferencia por la definición usada para hipotensión. En un estudio realizado en la población obstétrica del Hospital Universitario de Santander, se encontró que la incidencia de HBAS fue del $25,76 \%$ en paciente llevadas a Cesárea, usando bajas dosis de anestésico local ${ }^{8}$.

Se han diseñado múltiples estrategias encaminadas a reducir de forma significativa la frecuencia de presentación de hipotensión, el colocar a la gestante en decúbito lateral izquierdo para disminuir la compresión aortocava9 ${ }^{9}$, el empleo de bolos de líquidos intravenosos ${ }^{10-12}$; la reducción de la dosis de anestésico ${ }^{13}$, la elevación o compresión de los miembros inferiores ${ }^{9}$ y la administración de vasopresores ${ }^{14-16}$, son ejemplos de ello, con resultados contradictorios en su eficacia ${ }^{17}$. En los últimos años, ha surgido un interés particular por la administración de fenilefrina profiláctica, dado su principal efecto a adrenérgico ${ }^{18-20}$, sin embargo, los estudios realizados ${ }^{21,23}$ no han determinado la dosis o la forma de administración que resulte eficaz en la prevención de este evento adverso.

Lo contradictorio de los resultados sobre la administración profiláctica de fenilefrina, sumado a la ausencia de reportes con adecuado nivel de evidencia acerca de su uso en nuestro medio, nos motivó a plantear un Ensayo Clínico Controlado (ECC) aleatorizado con placebo administrando Fenilefrina a una tasa de infusión fija, con el objetivo de evaluar su eficacia profiláctica en la prevención de la hipotensión inducida por anestesia subaracnoidea.

\section{Materiales y métodos}

Se realizó un Ensayo Clínico Controlado (ECC) aleatorizado con placebo de solución salina normal versus administrando fenilefrina a una tasa de 
infusión fija. Este estudio contó con la aprobación del comité de ética de la Clínica Materno Infantil San Luis en Bucaramanga, durante los meses de junio a noviembre de 2016 y se registró en www. clinicaltrials.gov bajo el código NCT02979405. Para el cálculo del tamaño muestral, se consideraron estudios previos a nivel mundial, con reportes de incidencia de hipotensión tan altos como $70 \%$ y valores inferiores a un $20 \%$, por lo que se decidió realizar en promedio entre $40 \%$ y $20 \%$ y deseando encontrar una disminución de esta incidencia en un $50 \%$. Usando una confianza del $95 \%$ y poder del $80 \%$. Según la Fórmula Fleiss, se requerían 64 pacientes por grupo más el ajuste del 10\% considerando las posibles pérdidas, dió 70 pacientes por grupo, para un total de 140 pacientes.

Se incluyeron en estudio pacientes mayores de 18 años, clasificación American Society of Anesthesiologists (ASA) 1 y 2, con edad gestacional mayor a 37 semanas, quienes fueron llevadas a cesárea y cuya técnica anestésica fue anestesia subaracnoidea. Se excluyeron pacientes con trastornos hipertensivos asociados al embarazo, sufrimiento fetal agudo, embarazo gemelar, antecedentes de enfermedad cerebrovascular o cardiovascular, hipersensibilidad a la fenilefrina o rechazo para participar en estudio. Se obtuvo consentimiento informado de todas las pacientes ${ }^{24-27}$. Se diseñaron dos grupos de estudio, un grupo que recibió la intervención y otro considerado control. La aleatorización fue realizada mediante una tabla generada por computador en bloques. Tanto la paciente como el anestesiólogo a cargo se encontraban enmascarados.

Las mezclas fueron preparadas por una central de mezclas institucional. El grupo intervenido recibió una infusión de $40 \mathrm{mcg} / \mathrm{min}$ de fenilefrina, por 5 minutos, iniciando desde la administración del anestésico local; el grupo control recibió 10 cc de solución salina normal. Todas las pacientes por protocolo institucional, fueron canalizadas con bránula $\mathrm{N}^{\circ} 16 \mathrm{G}$ y se inició una infusión de lactato de ringer. A su ingreso a la sala quirúrgica, se les colocó monitoreo no invasivo estándar, incluyendo monitoreo electrocardiográfico, pulso oximetría y se tomó la tensión arterial cada minuto durante 3 minutos para calcular la tensión arterial basal. La anestesia subaracnoidea fue administrada a todas las pacientes sentadas, con Pencan ${ }^{\circledR}$ (B Braun Medical, Inc.) $25 \mathrm{G}$ bupivacaína pesada $10 \mathrm{mg}+$ fentanilo 20 mcg + morfina $80 \mathrm{mcg}$, durante 30 segundos. Una vez finalizó la administración de la anestesia, se inició la infusión del medicamento mediante bomba de infusión por cinco minutos. Se retornó la paciente a la posición supino, con desviación uterina y se aumentó la tasa de infusión de lactato de ringer.

Por personal entrenado en la ejecución del protocolo, se realizaron tomas de tensión arterial, frecuencia cardíaca y saturación de oxígeno cada minuto, en los primeros 5 minutos y posteriormente cada 5 minutos hasta su traslado a sala de recuperación. Se midió el nivel sensitivo al minuto 5, 10 y 15 mediante pruebas de sensibilidad térmica. El desenlace primario evaluado durante todo el procedimiento fue hipotensión arterial, definido como TAS $<90 \mathrm{mmHg}$ o TAM $<60 \mathrm{mmHg}$ o ambas, medido mediante monitor no invasivo. Otras variables que se valoraron en las pacientes fueron edad, peso y talla, escolaridad, presencia de náusea y vómito durante el procedimiento, APGAR neonatal al minuto 1 y 5 y requerimiento de dosis adicionales de vasopresores durante el procedimiento. No se administró profilaxis antiemética.

\section{Análisis estadístico}

Los datos se registraron en una base de datos en Excel, la cual se llevó al análisis final en STATA ${ }^{\circledR}$ 14. Las características clínicas de las pacientes se describieron usando medias y proporciones con su respectivo intervalo de confianza al $95 \%$. El test de la $t$ y el test exacto de Fischer con su significancia al 0,05 se usó para evaluar las diferencias entre las variables continuas y dicotómicas respectivamente.

El Riesgo Relativo (RR) con Intervalo de Confianza al 95\% (IC 95\%) de hipotensión y de otros eventos adversos se estimó para cada grupo de tratamiento. Este valor se usó para medir la eficacia de la intervención comparada con el grupo control. Se realizó análisis univariado, bivariado y, regresión logística, esta última con el fin de evaluar los efectos independientes de los posibles factores de riesgo clínico para la presencia de hipotensión. Un valor $\mathrm{p}$ $<0,05$ fue considerado significativo. Finalmente se realizó bondad de ajuste del modelo, con el fin de evaluar si los datos encontrados se relacionan con los esperados.

\section{Resultados}

Se consideraron elegibles 229 pacientes, de estas 140 pacientes fueron incluidas en el estudio (Ver Figura 
1). No hubo pérdidas en el estudio. No se encontraron diferencias significativas entre las características sociodemográficas, el nivel sensitivo logrado, el tiempo quirúrgico o el sangrado intraoperatorio de ambos grupos (Ver Tabla 1).

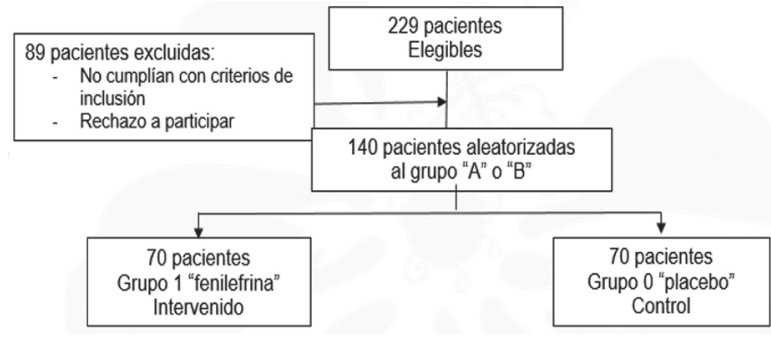

Figura 1. Reclutamiento de pacientes en el estudio. Fuente: autores

Tabla 1. Características basales de las pacientes

\begin{tabular}{|c|c|c|c|}
\hline \multirow[t]{2}{*}{ Características } & $\begin{array}{c}\text { Grupo } \\
\text { intervenido } \\
\mathrm{N}=\mathbf{7 0}\end{array}$ & $\begin{array}{l}\text { Grupo control } \\
\qquad N=70\end{array}$ & \multirow{2}{*}{$\begin{array}{l}\text { Valor } \\
\text { de P }\end{array}$} \\
\hline & $\begin{array}{c}\text { Media } \\
\text { DS (Min-Max) }\end{array}$ & $\begin{array}{c}\text { Media } \\
\text { DS (Min-Max) }\end{array}$ & \\
\hline Edad (años) & $\begin{array}{c}27,9 \\
6,2(18-43)\end{array}$ & $\begin{array}{c}29 \\
6,5(18-45)\end{array}$ & 0,3357 \\
\hline Peso (Kg) & $\begin{array}{c}72,5 \\
10,9(48-101)\end{array}$ & $\begin{array}{c}73,0 \\
9,4(48-95)\end{array}$ & 0,7653 \\
\hline IMC & $\begin{array}{c}28,4 \\
3,7(20,5-37,1)\end{array}$ & $\begin{array}{c}28,3 \\
3,6(20-36,1)\end{array}$ & 0,9593 \\
\hline & $\%(\mathrm{Fr})$ & $\%(\mathrm{Fr})$ & \\
\hline Escolaridad & & & 0,493 \\
\hline Primaria & $42,86(3)$ & $57,14(4)$ & \\
\hline Secundaria & $54,8(40)$ & 45,2 (33) & \\
\hline Universitaria & $45(27)$ & $55(33)$ & \\
\hline ASA 1 & $52,4(22)$ & $47,6(20)$ & 0,712 \\
\hline ASA 2 & $49(48)$ & $51(50)$ & \\
\hline Urgencia & $50(34)$ & $50(34)$ & 1 \\
\hline Nivel Sensitivo & & & 0,865 \\
\hline T3-T4 & $50,8(32)$ & $49,2(31)$ & \\
\hline T5-T8 & 49,4 (38) & $50,6(59)$ & \\
\hline $\begin{array}{l}\text { Tiempo } \\
\text { Quirúrgico }\end{array}$ & $\begin{array}{c}56 \\
9(35-80)\end{array}$ & $\begin{array}{c}55 \\
8,3(40-80)\end{array}$ & 0,5077 \\
\hline $\begin{array}{l}\text { Líquidos } \\
\text { administrados }\end{array}$ & $\begin{array}{c}2371 \\
(265 ; 1500-3000)\end{array}$ & $\begin{array}{c}2401 \\
(239 ; 1500-3000)\end{array}$ & 0,4823 \\
\hline $\begin{array}{l}\text { Sangrado } \\
\text { intraoperatorio }\end{array}$ & $\begin{array}{c}676 \\
(80 ; 500-1000)\end{array}$ & $\begin{array}{c}672 \\
(73 ; 400-800)\end{array}$ & 0,784 \\
\hline
\end{tabular}

Fuente: autores.
El monitoreo de todas las variables hemodinámicas se realizó hasta la terminación del acto anestésico quirúrgico. Durante los primeros 40 minutos en ambos grupos permanecieron las 70 pacientes, luego el tamaño empezó a disminuir progresivamente,según la duración del procedimiento quirúrgico, hasta 35 y 30 pacientes en el grupo control y el intervenido respectivamente al minuto 60 , que fue la máxima duración del procedimiento. El comportamiento hemodinámico de las pacientes evidenció el promedio por cada grupo y en cada momento evaluado nunca estuvo con valores definidos como hipotensión ya fuese para TAS o TAM, pero siempre fueron menores y significativos en el grupo control en los intervalos entre el minuto $2 \mathrm{y}$ el minuto $10 \mathrm{y}$ posteriormente al minuto 20 (Ver Figura 2).

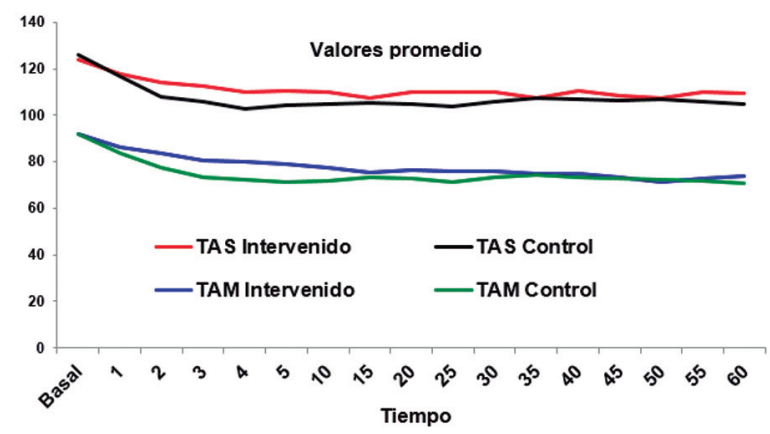

TAS: Tensión arterial sistólica, TAM: tensión arterial media Figura 2. Comportamiento hemodinámico según grupo de estudio. Fuente: autores.

En el análisis de los resultados del comportamiento hemodinámico, según las definiciones de hipotensión, se encontró que el grupo control presentó mayor incidencia (TAS 45,71 en comparación con 32,85\% $p=0,119$, TAM: 44,29 en comparación con 41,43 $p=0,733)$.

Al evaluar el momento en el cual se presentó la hipotensión, se evidenció que en el grupo control, entre los minutos 3 y 10 se presentó su más alta incidencia, con diferencias significativas en el minuto 4, minuto 5 y minuto 20. En el grupo intervenido la mayor incidencia de hipotensión se presentó en el minuto 20 (12,86\%), donde fue mayor que en el grupo control, seguido de los minutos 3 y 10 , siendo estos valores menores que en el grupo control (Ver Figura 3).

La incidencia de náuseas, vómito, hipertensión, bradicardia y requerimientos adicionales de fenilefrina, encontrando diferencias estadísticamente significativas entre los grupos, en la presentación de 
náuseas, náuseas y vómito y el requerimiento de vasopresores adicional al de estudio (Ver Tabla 2). No se encontraron diferencias significativas en el puntaje de APGAR entre los grupos de estudio.

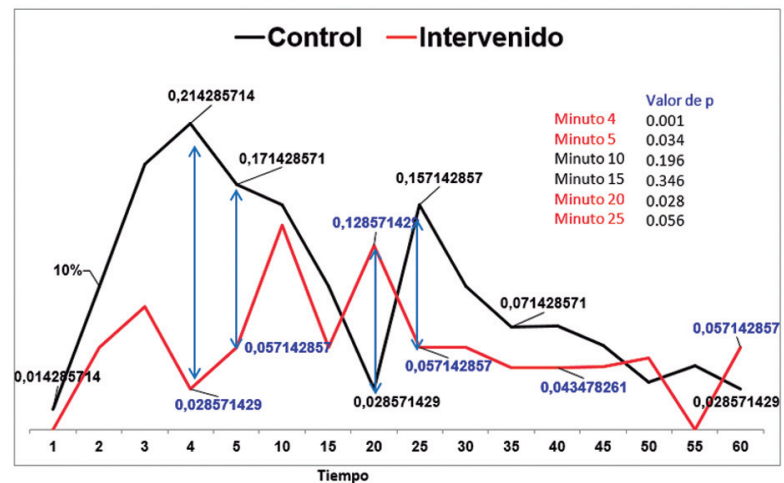

Figura 3 Incidencia de Hipotensión Arterial Sistólica según momento Fuente: autores

Tabla 2. Riesgos Absolutos y Relativos de eventos adversos según grupos

\begin{tabular}{|c|c|c|c|c|}
\hline & \multicolumn{2}{|c|}{ Riesgo Absoluto } & $\begin{array}{l}\text { Riesgo } \\
\text { Relativo }\end{array}$ & \\
\hline \multirow{2}{*}{$\begin{array}{l}\text { Desenlaces } \\
\text { secundarios }\end{array}$} & \multicolumn{2}{|c|}{ Grupo \% (Fr) } & \multirow{2}{*}{$\begin{array}{l}\text { Control Vs } \\
\text { Intervenido }\end{array}$} & \multirow{2}{*}{$\begin{array}{c}\text { Valor } \\
p\end{array}$} \\
\hline & Control & Intervenido & & \\
\hline Náuseas & $52,86(37)$ & 34,29 (33) & $\begin{array}{c}1,54(1,04- \\
2,3) \\
\end{array}$ & 0,027 \\
\hline Vómito & $15,71(11)$ & $7,14(5)$ & $2,2(0,8-6,0)$ & 0,111 \\
\hline $\begin{array}{l}\text { Náusea y } \\
\text { Vómito }\end{array}$ & $17,14(12)$ & $5,71(4)$ & $3(1,02-8,9)$ & 0,034 \\
\hline Bradicardia & $32,86(23)$ & $31,43(22)$ & $\begin{array}{c}1,05(0,65- \\
1,70) \\
\end{array}$ & 0,856 \\
\hline Hipertensión & $1,43(1)$ & $2,86(2)$ & $0,5(0,05-5,4)$ & 0,559 \\
\hline $\begin{array}{l}\text { Requerimiento } \\
\text { de vasopresor }\end{array}$ & $61,5(32)$ & $38,5(20)$ & $\begin{array}{c}1,4(1,02- \\
1,86)\end{array}$ & 0,032 \\
\hline
\end{tabular}

Fuente: autores

\section{Discusión}

La hipotensión bajo anestesia subaracnoidea en cesárea es uno de los principales eventos adversos relacionados directamente con el acto anestésico, ${ }^{4,5}$, asociándose a importantes complicaciones tanto maternas como fetales. El objetivo de los estudios actuales está enfocado en la búsqueda del vasopresor ideal, que evite la aparición de hipotensión, considerándose el uso de la efedrina ${ }^{14,15}$, fenilefrina ${ }^{16-23}$, etilefrina. En la actualidad fenilefrina es el más usado ${ }^{17}$, pero no se ha podido establecer la forma ideal de administración, la dosis requerida y su eficacia significativa en la reducción de la frecuencia de presentación de hipotensión.

Los resultados obtenidos en este estudio son concordantes con lo reportado por Allen et al., en donde al evaluar 4 diferentes tasas de infusión fijas de fenilefrina profiláctica, comparado con placebo, no se encontró una diferencia estadísticamente significativa en la reducción de la hipotensión ${ }^{22}$, aunque en nuestro estudio, la incidencia de hipotensión incluso en el grupo control, fue menor que la reportada en otros trabajos, quizá esto relacionado con la dosis total de bupivacaína pesada (10 mg) administrada, al compararse con otros estudios $(12,5-15 \mathrm{mg})^{22,24}$.

Si bien, la eficacia profiláctica de la fenilefrina en disminuir la incidencia de forma significativa de hipotensión no se evidenció, los hallazgos nos permitieron documentar una diferencia significativa en los requerimientos de vasopresores entre las pacientes que presentaron hipotensión durante el procedimiento quirúrgico, a favor del grupo que recibió la intervención, lo cual se ha reportado en la literatura 22,24 .

Adicionalmente, se evidenció una reducción significativa en la presentación de náusea y vómito en el grupo intervenido, a pesar de que el diseño del estudio ni el cálculo del tamaño muestral fue realizado para evaluar estos síntomas; los resultados son congruentes con lo reportado en la literatura9, considerándose un factor protector, aún cuando en la mezcla anestésica se administraron dos opioides (morfina, fentanilo), los cuales pueden incrementar el riesgo de presentar estos eventos.

No evidenciamos diferencias significativas en cuanto a la presencia de efectos adversos asociados al uso de fenilefrina como bradicardia e hipertensión, las cuales se presentaron de forma frecuente en estudios que usaban dosis iguales o mayores a $100 \mathrm{mcg} / \mathrm{min}$ de este medicamento ${ }^{22}$, ya que, en el presente estudio, se usó una dosis fija y considerada en la literatura, como baja ${ }^{21,22}$, confirmando el perfil de seguridad para su uso, en este tipo de pacientes. Adicionalmente, se encontró que en el grupo intervenido la hipertensión se relacionó en algunos casos con la administración de vasopresores adicionales, para manejo de episodios de hipotensión, de lo cual se podría inferir que en las pacientes que se usa fenilefrina profiláctica, las dosis requeridas de vasopresores para tratar la hipotensión cuando esta se llegase a 
presentar, pueden ser menores respecto de las dosis usadas en pacientes que no reciben profilaxis.

Aunque no se evaluó en el estudio el impacto hemodinámico de la oxitocina, que, asociado con la dosis y rapidez de la administración ${ }^{28}$, puede llegar a ser importante, este no era uno de los objetivos de este estudio, además, no se consideró significativo, ya que en la institución donde se realizó la recolección de las pacientes, existe un protocolo de administración, por lo que no fue un factor diferencial en los grupos de estudio.

\section{Conclusiones}

La fenilefrina profiláctica, en dosis de $40 \mathrm{mcg} / \mathrm{min}$, no fue eficaz para disminuir la incidencia de hipotensión arterial inducida por la anestesia subaracnoidea en gestantes llevadas a cesárea. El presentar riesgos absolutos y relativos menores pero no significativos de presentar hipotensión en el grupo intervenido, podría permitir realizar estudios posteriores, recalculando el tamaño muestral, para que los valores obtenidos en el estudio, puedan tener significancia estadística.

Sin que haya sido el objetivo del estudio, el requerimiento total de vasopresor presentó diferencia significativa entre los grupos, con un RR mayor en el grupo control, pudiendo considerarse como posibilidad, que si ajustáramos la dosis o el tiempo de la infusión profiláctica, esto nos permitiría poder en un subsiguiente estudio demostrar la eficacia y disminuir las intervenciones realizadas para su manejo.

La frecuencia de eventos adversos como náuseas y vómito evaluados en el intraoperatorio, al ser menores y significativo en el grupo intervenido, pudiéramos considerarlas como un potencial efecto protector, pues la incidencia de estos eventos fue muy similar a la de hipotensión y un estudio posterior con un cálculo para evaluar estas diferencias entre grupos nos permitiría obtener el beneficio adicional de la fenilefrina ante la aparición de estos síntomas que podríamos considerarlos como un evento adverso secundario a la hipotensión cuando se presenta en los primeros minutos.

Aún no tenemos el gold estándar en el manejo profiláctico de la hipotensión ${ }^{23,29}$, pero se abren nuevas alternativas, que pudieran no ser nuevos fármacos, sino ajustes en la dosis y el tiempo de su aplicación, considerando que la anestesia regional subaracnoidea en cesárea seguirá siendo una técnica a elegir.

\section{Referencias bibliográficas}

1. World Health Organization. Appropriate Technology for Birth. Lancet. 1985;326(8452):436-7.

2. Rubio-Romero JA, Fonseca-Pérez JE, Molina S, Buitrago-Leal M, Zuleta JJ, Ángel-Müller E, et al. Racionalización del uso de la cesárea en Colombia. Consenso de la Federación Colombiana de Obstetricia y Ginecología (FECOLSOG) y la Federación Colombiana de Perinatología (FECOPEN). Bogotá, 2014. Rev Colomb Obstet Ginecol . 2014;65(2):139-51.

3. Williams JW. Obstetrics: A Texbook for the Use of Students and Practitioners. New York : Appleton and Company.

4. Chestnut DV, Wong CA, Tsen L, Ngan Kee W, Beilin Y, Mhyre J. Chestnut's Obstetric Anesthesia: Principles and Practice. Philadelphia: Elsevier; 2014.

5. Miler, RD. Miller's Antesthesia. 8a ed. España: Elsevier; 2016.

6. KlÖhr S, Roth R, Hofmann T, Rossaint R, Heesen M. Definitions of hypotension after spinal anaesthesia for caesarean section: Literature search and application to parturients. Acta Anaesthesiol Scand. 2010;54(8):909-21

7. Mavridou I, Stewart A, Fernando R. Maternal Hypotension During Spinal Anesthesia for Cesarean Delivery. Curr Anesthesiol Rep. 2013;3(4):282-91.

8. Chooi C, Cox JJ, Lumb RS, Middleton P, Chemali M, Emmett RS, et al. Techniques for preventing hypotension during spinal anaesthesia for caesarean section. Cochrane Database Syst Rev. 2010;(3):11

9. Ouerghi S, Bougacha MA, Frikha N, Mestiri T, Ben Ammar MS, Mebazaa MS. Combined use of crystalloid preload and low dose spinal anesthesia for preventing hypotension in spinal anesthesia for cesarean delivery: A randomized controlled trial. Middle East J Anesthesiol. 2010;20(5):667-72.

10. Dyer RA, Farina Z, Joubert IA, Du Toit P, Meyer M, Torr G, et al. Crystalloid preload versus rapid crystalloid administration after induction of spinal anesthesia for elective cesarean section. Anaesth Intensive Care. 2004;32(3):351-7.

11. Banerjee A, Stocche RM, Angle P, Halpern SH. Preload or coload for spinal anesthesia for elective Cesarean delivery: A metaanalysis. Can J Anaesth. 2010;57(1):24-31.

12. Arzola C, Wieczorek PM. Efficacy of low-dose bupivacaine in spinal anaesthesia for Caesarean delivery: systematic review and meta-analysis. Br J Anaesth. 2011;107(3):308-18.

13. Gupta S. Vasopressors and tight control of maternal blood pressure during cesarean delivery: A rocky Alliance. J Anaesthesiol Clin Pharmacol. 2013;29(1):1-3.

14. Dyer RA, Biccard BM. Ephedrine for spinal hypotension during elective caesarean section: the final nail in the coffin? Acta Anaesthesiol Scand. 2012;56(7):807-9.

15. Ngan Kee WD, Khaw KS, Tan PE, Ng FF, Karmakar MK. Placental transfer and fetal metabolic effects of phenylephrine and ephedrine during spinal anesthesia for cesarean delivery. Anesthesiology. 2009;111(3):506-12.

16. Heesen M, Klöhr S, Rossaint R, Straube S. Prophylactic phenylephrine for caesarean section under spinal anaesthesia: Systematic review and meta-analysis. Anaesthesia. 2014;69(2):143-65.

17. Habib AS. A review of the impact of phenylephrine administration on maternal hemodynamics and maternal and neonatal outcomes in women undergoing cesarean delivery under spinal anesthesia. Anesth Analg. 2012;114(2):377-90.

18. Stewart A, Fernando R, McDonald S, Hignett R, Jones T, Columb $\mathrm{M}$. The dose-dependent effects of phenylephrine for elective cesarean delivery under spinal anesthesia. Anesth Analg. 2010;111(5):1230-7.

19. Mahajan L, Anand LK, Gombar KK. A randomized doubleblinded comparison of ephedrine, phenylephrine and mephentermine infusions to maintain blood pressure during spinal anaesthesia for cesarean delivery: the effects on fetal acid-base status and haemodynamic control. J anaesthesiol clin 
pharmacol. 2009;25(4):427-32.

20. Ngan WW, Khaw K, Ng FF. Prevention of hypotension during spinal anesthesia for cesarean delivery. an effective technique using combination phenylephrine infusion and crystalloid cohydration. Anesthesiology. 2005;103(4):744-50.

21. Allen TK, George RB, White WD, Muir HA, Habib AS. A double-blind, placebo-controlled trial of four fixed rate infusion regimens of phenylephrine for hemodynamic support during spinal anesthesia for cesarean delivery. Anesth Analg. 2010;111(5):1221-9.

22. Ngan Kee. Phenylephrine Infusions for Maintaining Blood Pressure During Spinal Anesthesia for Cesarean Delivery: Finding the Shoe That Fits. Anesth Analg. 2014;118(3):496-498

23. Doherty A, Ohashi Y, Downey K, Carvalho J. Phenylephrine Infusion Versus Bolus Regimens During Cesarean Delivery Under Spinal Anesthesia: A Double-Blind Randomized Clinical Trial to Assess
Hemodynamic Changes. Anesth Analg. 2012;115(6):1343-1350.

24. World Medical Association. World Medical Association Declaration of Helsinki-Ethical principles for medical research involving human subjects. JAMA. 2013;310(20):2191-4.

25. Resolución 8430 de 1993. Colombia. Ministerio de Salud. 1993:1-19.

26. Ley estatutaria 1581 de 2012. Colombia. Congreso de la República. 2012.

27. Pinder AJ, Dresner M, Clow C, Shorten GD, O’Riordan J, Johnson R. Haemodynamic changes caused by oxytocin during caesarean section under spinal anaesthesia. Int J Obstet Anesth. 2002;11 (3):156-15.

28. American Society of Anesthesiologists. Practice Guidelines for Obstetric Anesthesia. ASA. 2016;24(2):270-300.

29. SOAP. Practice Guidelines for Obstetric Anesthesia. Vol. 124, Anesthesiology. 2016. 270-300 